\title{
Aconselhamento nutricional de crianças menores de dois anos de idade: potencialidades e obstáculos como desafios estratégicos
}

\author{
Nutritional counseling for children under two years of age: \\ opportunities and obstacles as strategic challenges
}

\author{
Aline Aparecida de Oliveira Campos ${ }^{1}$ \\ Rosângela Minardi Mitre Cotta ${ }^{1}$ \\ Julicristie Machado de Oliveira ${ }^{2}$ \\ Adriana Kelly Santos ${ }^{3}$ \\ Raquel Maria Amaral Araújo ${ }^{1}$
}

${ }^{1}$ Departamento de Nutrição e Saúde, Universidade Federal de Viçosa. Av. P H Rolfs S/N, Centro. 36.571-000 Viçosa MG Brasil.

alinejsc@yahoo.com.br ${ }^{2}$ Faculdade de Ciências Aplicadas, Universidade

Estadual de Campinas.

${ }^{3}$ Laboratório de

Comunicação e Saúde, Instituto de Comunicação e Informação Científica e Tecnológica em Saúde, Fundação Oswaldo Cruz.
Abstract This article sought to conduct a critical analysis of the state of the art of the scientific production in counseling practices in nutrition for infants under two years of age, discussing the opportunities and obstacles in terms of strategic challenges for implementing healthy nutrition practices. It is a bibliographic study, with the core interest being counseling on infant nutrition. The majority $(58.1 \%)$ of the articles analyzed was published between 2008 and 2011. The educative actions of counseling on infant feeding were directed at health professionals and caregivers to children under two years of age. As opportunities in the practice of nutritional counseling the following stand out: an improvement in the performance of health professionals, the adoption of healthy eating practices by caregivers, and an improvement in the nutritional status of the children. As obstacles, the following stand out: a lack of training of caregivers and professionals regarding complementary healthy feeding and difficulties regarding the practice of counseling by a health care professional. This evidence reaffirms the importance of incorporating counseling guidance on infant feeding, as permanent practices in policies for child health care.

Key words Counseling, Complementary feeding, Breastfeeding
Resumo Este artigo teve como objetivo analisar criticamente o estado da arte da produção científica sobre as práticas do aconselhamento em alimentação da criança menor de dois anos de idade, discutindo sobre as potencialidades e obstáculos enquanto desafios estratégicos para a implementação de práticas de alimentação saudável. Trata-se de um estudo de natureza bibliográfica, tendo como núcleo de interesse o aconselhamento em alimentação infantil. A maioria $(58,1 \%)$ dos artigos analisados foi publicada entre 2008 e 2011. As ações educativas de aconselhamento em alimentação infantil foram direcionadas aos profissionais de saúde e aos cuidadores de crianças menores de dois anos de idade. Como potencialidades da prática do aconselhamento nutricional destacam-se: melhora no desempenho dos profissionais de saúde, adoção de práticas alimentares saudáveis pelos cuidadores e melhora no estado nutricional das crianças. Como obstáculos, ressalta-se: falta de capacitação dos cuidadores e profissionais quanto à alimentação complementar saudável e dificuldade quanto à prática do aconselhamento por profissionais de saúde. Essas evidências reafirmam a importância da incorporação de ações de aconselhamento em alimentação infantil, como práticas permanentes, às políticas de atenção à saúde da criança.

Palavras-chave Aconselhamento, Alimentação complementar, Aleitamento materno 


\section{Introdução}

As recomendações atuais sobre alimentação infantil saudável ressaltam a importância do aleitamento materno exclusivo durante os seis primeiros meses de vida e a continuidade da amamentação, após o início da alimentação complementar, até os dois anos de idade ou mais ${ }^{1-3}$.

Neste contexto, a orientação nutricional da criança assume papel imprescindível para garantir um desenvolvimento satisfatório ${ }^{4}$ introduzindo os alimentos adequadamente, propiciando à criança meios para que atinja o seu potencial biológico $^{5,6}$. Diante a importância em se orientar adequadamente cuidadores de crianças menores de dois anos de idade e profissionais de saúde quanto à prática da alimentação infantil, justifica-se a retomada dos estudos do aconselhamento nutricional, conceituado, em 1969, pela Associação Americana de Dietética, como a orientação profissional individualizada, com a finalidade de aconselhar as pessoas quanto à prática da alimentação saudável, a fim de se atender às necessidades nutricionais ${ }^{7}$. Neste sentido, o aconselhamento nutricional se mostra como importante estratégia educativa que precisa ser resgatada, a fim de redimensionar a atuação do nutricionista junto ao indivíduo que necessita mudar e/ou reorientar seu comportamento alimentar ${ }^{8}$.

Estudos mostram que a aconselhamento nutricional é uma excelente estratégia na (re)construção dos hábitos alimentares e promoção de estilos de vida saudáveis ${ }^{9-11}$, proporcionando, além de conhecimentos acerca da alimentação, mudanças comportamentais significativas que melhoram a qualidade de vida da população $0^{8,12,13}$. Trabalho realizado por Santos et al. ${ }^{14}$ revelou que o aconselhamento nutricional oferecido após capacitação dos profissionais de saúde associou-se à melhora no desempenho das práticas maternas, bem como ao crescimento infantil. Bassichetto e Rea ${ }^{15}$ destacam a importância de se incorporar atividades de aconselhamento em alimentação infantil às políticas de atenção à saúde da criança como práticas contínuas, reafirmando, portanto, a preocupação com a educação permanente. Os cursos de aconselhamento promovidos pela Organização Mundial da Saúde (OMS) propõem o uso de técnicas que podem facilitar a comunicação, estimulando assim, o processo de educação. Estudos já apontaram vantagens de capacitações na melhoria das práticas das mães e dos profissionais de saúde com relação à nutrição e alimentação infantis ${ }^{14,16}$.
Desde esta perspectiva, o presente estudo tem como objetivo analisar criticamente o estado da arte da produção científica sobre as práticas do aconselhamento em alimentação da criança menor de dois anos de idade, discutindo sobre as potencialidades e obstáculos enquanto desafios estratégicos para a implementação de práticas de alimentação saudável.

\section{Métodos}

Trata-se de um estudo de natureza bibliográfica, realizado por meio de uma revisão minuciosa da literatura científica, entre os meses de setembro a novembro de 2011, tendo como núcleo de interesse o aconselhamento em alimentação da criança menor de dois anos de idade. As informações sobre os estudos foram extraídas das revistas indexadas nas bases de dados em saúde Lilacs (Literatura Latino-americana e do Caribe), Medline (Medical Literature Analysis and Retrieval System Online), e SciELO (Scientific Eletronic Library Online). Os termos utilizados nas buscas bibliográficas foram aconselhamento, aconselhamento nutricional, orientação nutricional, aleitamento materno, alimentação complementar e alimentação infantil.

No intuito de contemplar toda a produção científica referente às práticas do aconselhamento nutricional em alimentação da criança menor de dois de idade, o período de publicação não foi delimitado, sendo encontrados artigos publicados entre os anos de 1998 e 2011.

A busca inicial resultou em 1541 artigos que abordavam questões referentes ao aconselhamento em alimentação infantil. Em um primeiro momento, excluindo-se os repetidos por bases de dados, resultaram 1182 artigos. Posteriormente, foi realizado um refinamento a partir da leitura dos títulos e resumos a fim de selecionar os artigos relacionados à temática em questão, que foram lidos integralmente. Foram desconsideradas publicações cujos dados haviam sido coletados em outros países, sendo, portanto incluídos os artigos que apresentavam dados coletados no Brasil e revisões relevantes acerca do aconselhamento nutricional em alimentação da criança menor de dois de idade, totalizando, ao final, 29 artigos que foram analisados integralmente (Figura 1).

Após leitura sistemática dos estudos, as potencialidades e os obstáculos presentes referentes ao aconselhamento nutricional em alimentação de crianças menores de dois anos de idade foram agrupados em categorias (Tabelas 1 e 2). 
|́ Aconselhamento |

nutricional

( - - - - - - v

| Aconselhamento |

ᄂ $-\ldots-\ldots$

------ v

Orientação nutricional

( $\ldots+\ldots,-\ldots$

$--\cdots-\cdots$

Alimentação infantil

- - - - -

$----->$

Alimentação

complementar

' - - - - ,

------ ,

Aleitamento materno

- - - - - - - -

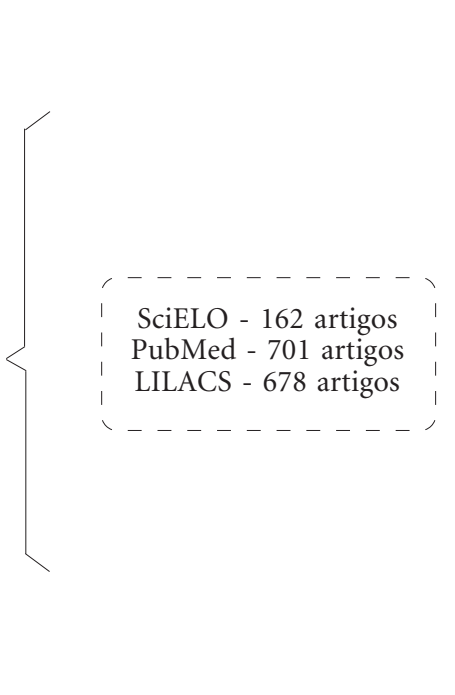

\}

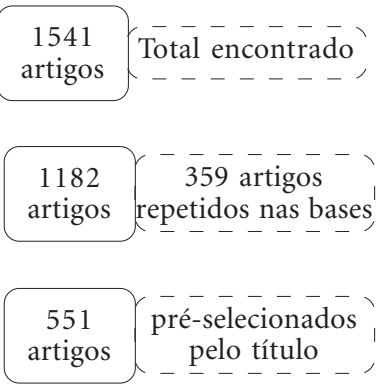

61 ( pré-selecionados artigos pelo resumo

29 artigos selecionados para análise integral

\section{Termos utilizados \\ Busca nas bases de dados \\ Processo de seleção}

Figura 1. Roteiro sistematizado para realização da pesquisa e seleção de artigos nas bases de dados do SciELO, Lilacs e Medline (2011).

Por potencialidades consideramos todos os aspectos que contribuíram positivamente para a prática do aconselhamento nutricional, contribuindo direta ou indiretamente, para a melhora da qualidade de vida da população em estudo. Por outro lado, foram considerados como obstáculo todo e qualquer aspecto que contribuiu negativamente para o adequado desenvolvimento e multiplicação desta prática.

\section{Resultados}

Os achados referentes à análise dos artigos apontam os originais correspondendo a 58,62\% ( $\mathrm{n}=$ 17) e os estudos de revisão totalizando $41,38 \%$ $(\mathrm{n}=12)$ das publicações. Observa-se um aumento no número de publicações referentes à temática abordada entre os anos 1998 a 2011, quando no periodo de 2008-2011 houve um expressivo aumento do interesse pela temática (Gráfico 1).

Com relação ao grupo específico das ações de aconselhamento nutricional, observa-se uma predominância de estudos com ações educativas direcionadas aos profissionais de saúde ${ }^{2,14,15,17-}$ ${ }^{32}$, seguidas dos que têm como foco os cuidadores de crianças menores de dois anos de ida$\mathrm{de}^{2,14,17,21,22,24,28,30,33-42}$.

Categorizando os achados em potencialidades da prática do aconselhamento nutricional em alimentação infantil, os estudos revelaram aspectos importantes, reforçando, portanto, a pertinência em se inserir práticas educativas em alimentação e nutrição. A análise dos estudos apontou como principais potencialidades da prática do aconselhamento nutricional: melhora no desempenho dos profissionais de saúde quanto à prática do aconselhamento, bem como aumento no tempo de aleitamento materno exclusivo, melhora no estado nutricional das crianças menores de dois anos de idade quando comparados com período anterior ao aconselhamento e satisfação materna quanto às orientações recebidas; conforme pode ser visualizado na Tabela 1.

Com relação aos obstáculos que permeiam a prática do aconselhamento nutricional infantil, 
os resultados nos forneceram dados importantes quanto ao direcionamento das ações dos serviços de saúde (Tabela 2). Dentre os principais aspectos observados, destaca-se a necessidade ainda existente - de se capacitar profissionais de saúde para a realização do aconselhamento nutricional da criança menor de dois anos de idade.

\section{Discussão}

O aconselhamento nutricional direcionado aos profissionais de saúde, mães e/ou cuidadores configura-se como estratégia fundamental para a melhoria das práticas alimentares infantis ${ }^{10,43}$. Iniciativas têm sido implementadas a fim de re- qualificar profissionais de saúde para o aconselhamento ${ }^{15}$. Dentre estas iniciativas, destacam-se o Curso de Aconselhamento em Amamentação ${ }^{44}$, com o objetivo de capacitar profissionais de saúde em habilidades específicas de aleitamento materno; o Curso Integrado de Aconselhamento em Alimentação Infantil ${ }^{45}$, com a finalidade de contribuir para a compreensão dos fatores que influenciam o aconselhamento, além de sistematizar as principais características que o aconselhador deve ter para tornar esta prática eficiente; a Estratégia Nacional para Alimentação Complementar Saudável (ENPACS ${ }^{46}$, instrumento de fortalecimento das ações de apoio e promoção à alimentação complementar no âmbito da Atenção Primária à Saúde (APS), além de incentivar a

Tabela 1. Potencialidades da prática do aconselhamento nutricional em alimentação infantil presentes nos estudos analisados (1998 a 2011).

\begin{tabular}{lc}
\hline \multicolumn{1}{c}{ Potencialidades da prática do aconselhamento nutricional } & Estudos \\
\hline $\begin{array}{l}\text { Melhora no desempenho do profissional de saúde quanto à prática do } \\
\text { aconselhamento nutricional }\end{array}$ & $2,14,15,20,22,24,25,29,30,32,41$ \\
Satisfação materna quanto às orientações recebidas & $14,19,27,33,34,35$ \\
Aumento do tempo de aleitamento materno exclusivo & $17,33,34,38$ \\
Adoção de práticas alimentares saudáveis pelos cuidadores & $2,14,22,27$ \\
Melhora no estado nutricional das crianças & $2,14,22,35,40$ \\
Incentivo ao aleitamento materno por meio do aconselhamento & $20,27,34,36$ \\
realizado por profissionais de saúde & $19,35,41$ \\
Melhora no acolhimento/vínculo entre cuidadores e profissionais, & \\
favorecendo a relação profissional de saúde versus cuidador & 15,33 \\
Melhora no desempenho das respostas dos cuidadores & 14 \\
Aconselhamento nutricional oferecido ao grupo controle após a pesquisa & 35 \\
Redução de hospitalizações após aconselhamento de nutrição & \\
\hline
\end{tabular}

Tabela 2. Obstáculos acerca da prática do aconselhamento nutricional em alimentação infantil segundo estudos presentes na literatura (1998 a 2011).

\begin{tabular}{lc}
\hline Obstáculos que permeiam a prática do aconselhamento nutricional & Estudos \\
\hline $\begin{array}{l}\text { Falta de capacitação dos cuidadores quanto à introdução precoce da } \\
\text { alimentação complementar. }\end{array}$ & $18,26,27,28,30,37,39,42$ \\
$\begin{array}{l}\text { Falta de capacitação dos profissionais de saúde quanto à prática da } \\
\text { alimentação complementar saudável. }\end{array}$ & $26,27,28,35,36,38$ \\
$\begin{array}{l}\text { Falta de capacitação dos cuidadores quanto à prática da alimentação } \\
\text { complementar saudável. }\end{array}$ & $18,26,28,30,42$ \\
$\begin{array}{l}\text { Dificuldade quanto à prática do aconselhamento por profissionais de } \\
\text { saúde (acolher/ouvir/conquistar confiança dos cuidadores) } \\
\text { Divulgação insuficiente de materiais educativos pelos órgãos oficiais. }\end{array}$ & 14,27 \\
\hline
\end{tabular}




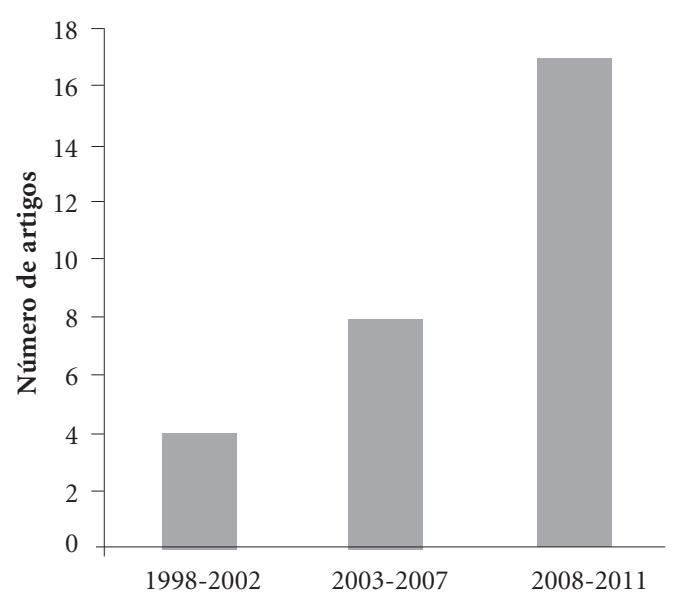

Gráfico 1. Publicações referentes ao aconselhamento nutricional em alimentação infantil (1998 a 2011).

orientação alimentar infantil como atividade de rotina nos serviços de saúde; e o Guia Alimentar para Crianças de 6 a 24 meses $^{47}$, uma iniciativa conjunta do Ministério da Saúde com a Organização Panamericana da Saúde, o qual, além de propor a pirâmide alimentar para essa faixa etária, traz os dez passos para uma alimentação saudável. Os guias alimentares são ferramentas práticas, por orientarem a população acerca da alimentação, através de informações de estímulo à amamentação e introdução saudável da alimentação complementar, incluindo orientações referentes à variedade e à consistência adequada dos alimentos. A evidência científica aponta para a importância estratégica dos guias alimentares enquanto importante instrumento para atender os objetivos dos programas de aconselhamento nutricional, por facilitarem a aprendizagem, contribuindo assim para uma adequada conduta alimentar $^{45,48,49}$.

No presente estudo, identificamos uma maior proporção de trabalhos cujo foco foi a capacitação dos profissionais de saúde. De acordo com Santos et al. ${ }^{14}$, a capacitação destes profissionais com vistas a prover as recomendações alimentares é fundamental quando se espera melhorar o conhecimento e o comportamento materno. É necessário, portanto, que o profissional de saúde coloque em prática as inúmeras experiências vivenciadas, possibilitando uma reflexão crítica e contextualizada dentro da realidade de cada família ${ }^{50}$.
Ainda em relação aos grupos específicos das ações de aconselhamento em alimentação infantil, a figura materna tem sido foco de inúmeros estudos. A mulher destaca-se, no contexto das políticas públicas de saúde, devido à sua característica de cuidadora e de maior preocupação com a saúde, tanto individual quanto familiar ${ }^{51-53}$. Segundo Correa et al..$^{54}$, os cuidados maternos são essenciais para a saúde da criança e podem sofrer influência da qualidade das informações em saúde, da escolaridade e idade materna, além do tempo de que a mãe dispõe para cuidar de seu filho. Romanelli55 reitera a importância deste grupo populacional no contexto da alimentação familiar por vários motivos, dentre eles por serem as principais responsáveis não somente pelo controle, mas também na quase totalidade dos domicílios pelo orçamento doméstico, compras e processamento dos alimentos que serão utilizados, além de socializarem os filhos a aceitá-los e distribuírem os alimentos entre os componentes da família. Aconselhamentos de qualidade para mães e cuidadores e mudanças de comportamento de outros familiares são essenciais para melhora das práticas alimentares infantis e deve estar no centro das estratégias destinadas a este fim ${ }^{10}$.

$\mathrm{O}$ aconselhamento orientado ao grupo materno infantil, cuja atenção se estenda desde o acompanhamento recebido pela gestante durante o pré-natal, enfatizando a importância da prática do aleitamento materno, passando pelo nascimento até o final da primeira infância, é recomendado como suporte às mães para a promoção da alimentação infantil adequada ${ }^{56,57}$. De acordo com Oliveira et al..$^{57}$, o aconselhamento nutricional promove a prática do aleitamento materno, além de contribuir para a introdução adequada da alimentação complementar, mesmo em grupos considerados vulneráveis, como o caso de mães adolescentes.

Assim, verifica-se a relevância de se realizar trabalhos de aconselhamento nutricional com as mães das crianças menores de dois anos, e não apenas realizar trabalhos cujo foco seja a criança, uma vez que, no contexto familiar de construção de hábitos alimentares, a figura materna tem-se mostrado fundamental para as escolhas alimentares da criança e consequentemente, da família como um todo ${ }^{39,58}$.

O aconselhamento em alimentação infantil é uma forma de atuação que permite a interação entre o profissional de saúde e a mãe, em um processo de escuta, no qual procura compreendê-la em seus diferentes anseios, permitindo além da multiplicação de conhecimentos, o planeja- 
mento das ações e a tomada de decisões por parte dela ${ }^{24,44,50}$.

No presente artigo, identificou-se as principais potencialidades da prática do aconselhamento, dentre eles a capacitação dos profissionais de saúde quanto às atividades direcionadas aos cuidadores das crianças menores de dois anos de idade, sobretudo as mães. O profissional de saúde deve apoiar as mães no processo de introdução de alimentos complementares, acolhendo-as e respeitando-as em suas particularidades, inquietações, dificuldades, conhecimentos prévios, êxitos e satisfações ${ }^{15}$. Neste sentido, a ENPACS mostra-se como estratégia efetiva para a promoção de hábitos alimentares saudáveis, uma vez que as oficinas direcionadas aos profissionais de saúde da APS habilitam os tutores em alimentação complementar, apoiando as famílias por meio do acompanhamento e orientação da alimentação saudável ${ }^{46}$. A fim de se obter informações a respeito das ENPACS e aperfeiçoar a sua implementação nos estados e municípios brasileiros, foram realizadas avaliações nas cidades de Santos e Brasília ${ }^{59}$. Os resultados apontaram que a metodologia crítico-reflexiva com enfoque problematizador adotada, permitiu a troca de experiências entre os diversos atores envolvidos nas oficinas de capacitação, possibilitando a construção do conhecimento a partir da realidade vivida por cada localidade. Ressaltou-se também a importância de se respeitar as crenças e saberes populares, e que estes devem ser levados em consideração ao se aconselhar as famílias em relação à prática da alimentação complementar saudável.

Rea e Venâncio ${ }^{16}$, em estudo realizado com profissionais de São Paulo, objetivando avaliar o Curso de Aconselhamento em Amamentação da OMS/UNICEF, mostraram melhora significativa em habilidades de aconselhamento. Porém, para que os mesmos passem a aplicar o aprendizado na prática, há necessidade de reforçar o manejo clínico da lactação e também de uma supervisão continuada. Bassichetto e Rea ${ }^{15}$ avaliaram o conhecimento e as práticas dos nutricionistas e médicos, submetidos ao Curso Integrado de Aconselhamento em Alimentação Infantil. Os resultados mostraram que o curso foi efetivo em aumentar os conhecimentos e as práticas de anamnese alimentar, porém não levou a uma melhora nas habilidades do aconselhamento, o que aponta para a necessidade de se implantar políticas de educação permanente, destacandose o papel estratégico das universidades na capacitação de profissionais dos serviços de saúde ${ }^{60}$.
Em estudo realizado em São Paulo, Mace$\mathrm{do}^{61}$, analisando a estratégia de capacitação em educação nutricional fornecida a educadores infantis de uma escola particular, encontrou aumento significativo no conhecimento destes após a intervenção, além de depoimentos de pais e alunos revelando mudanças positivas ocorridas no comportamento alimentar destes últimos.

Destarte, estudos têm focado a relação profissional-paciente como mediadora da qualidade do atendimento em saúde, incluindo iniciativas que incorporam habilidades de aconselhamento a fim de que o indivíduo se reconheça como sujeito de sua própria saúde e transformação ${ }^{62-64}$.

Estudo realizado por Gomes et al. ${ }^{65}$, em município de pequeno porte da Zona da Mata de Minas Gerais, objetivando avaliar o serviço realizado pelo Programa Saúde da Família, à partir das representações sociais dos entrevistados sobre as dimensões exclusivas da APS, identificou que a adesão de gestantes e nutrizes à prática do aleitamento materno é fortemente influenciada pelas orientações dos profissionais de saúde.

Pesquisa realizada em São Paulo e Recife ${ }^{66}$ identificou que os profissionais de saúde conheciam melhor a teoria do que a prática sobre aleitamento materno. Em uma amostra de 58 pediatras, cinco enfermeiros, 24 auxiliares e quatro atendentes prestadores de serviços das Unidades de Atenção Primária em Saúde da cidade de Campinas e região, pôde-se concluir que as orientações oferecidas pelos profissionais de saúde, de certa forma, refletiam àquelas recebidas durante o período de graduação e residência médica, sendo consideradas, portanto, como insuficientes em seu trabalho. Toma ${ }^{24}$ reitera que o conhecimento e o domínio da técnica a ser utilizada pelos profissionais de saúde, embora importantes, não são suficientes para o empoderamento das mulheres-mães quanto à prática adequada da alimentação dos seus filhos.

Embora os profissionais de saúde estejam sensibilizados quanto à importância do aleitamento materno e tenham conhecimentos teóricos sobre o tema, ainda apresentam dificuldades para resolução de questões práticas sobre o manejo da amamentação ${ }^{61}$. Em estudo realizado por Boog $^{67}$, médicos e enfermeiros relataram dificuldades para abordar as práticas alimentares, observando que a maioria dos médicos não estudou disciplinas que abordassem alimentação e nutrição, considerando, portanto, seus conhecimentos insuficientes. Estes achados vão ao encontro da pesquisa realizada por Silva et al. ${ }^{63}$, 
que em estudo sobre concepção de profissionais de saúde sobre alimentação saudável, realizado a partir de entrevistas e questionários com os mesmos, revelou que alimentação saudável é um tema que está muito disseminado, porém não se sentem capacitados para realizarem tais orientações. A prática da saúde coletiva seria enriquecida ao se adotar uma noção de cuidado que perpassa as competências técnicas, não se restringindo a elas $^{68}$. Segundo Toma ${ }^{24}$, a OMS, ao propor os cursos de aconselhamento em alimentação infantil, procura extinguir a distância entre a teoria apreendida e a prática existente. Estudos apontam os benefícios desses cursos de aconselhamento na melhoria das práticas das mães e dos profissionais de saúde com relação à nutrição $\mathrm{e}$ alimentação infantis ${ }^{16,63}$.

Ao enfatizar-se a importância dos aconselhamentos nutricionais para a multiplicação de hábitos alimentares saudáveis, não se pode esquecer que os comportamentos alimentares estão envoltos por um forte sistema de crenças $^{69}$, devendo, portanto, serem consideradas nas atividades de aconselhamento nutricional. Em estudo realizado por Mennella et al. ${ }^{70}$, a maioria das mães analisadas introduziu águas, chás e fórmulas infantis já na primeira semana de vida das crianças, embora estas mães já conhecessem os benefícios da amamentação exclusiva e a importância da adequada alimentação infantil, ficando evidente, portanto, a interferência das crenças, mitos e diferentes culturas nos comportamentos das mães em relação à alimentação de seus filhos. Ademais, ao se inserir práticas educativas de aconselhamento nutricional, o profissional deve estar consciente da importância de se basear na cultura alimentar da família, nos aspectos sociais e típicos da região, além dos mitos e crenças alimentares existentes ${ }^{70-72}$.

\section{Considerações finais}

A análise dos estudos publicados entre 1998 e 2011, sobre a prática do aconselhamento em alimentação da criança menor de dois anos de idade, apontou evidências que reafirmam a importância da incorporação de ações de aconselhamento em alimentação, como educação permanente dos profissionais de saúde e as políticas de atenção à saúde da criança.

O presente estudo evidenciou que, embora os obstáculos ainda existam e necessitem ser cuidadosamente trabalhados, a fim de se aprimorar o aconselhamento em alimentação da criança menor de dois anos de idade, as potencialidades presentes nesta prática superam esses entraves, evidenciando, portanto, a importância em inseri-las nas ações e serviços de saúde.

Destarte, compreender as potencialidades e os obstáculos que permeiam a prática do aconselhamento nutricional da criança menor de dois de idade é primordial quando se busca alternativas que visem aperfeiçoar a situação desta no país.

\section{Colaboradores}

AAO Campos, RMM Cotta, JM Oliveira, A KellySantos e RMA Araújo participaram igualmente de todas as etapas do artigo. 


\section{Referências}

1. Brasil. Ministério da Saúde (MS). Secretaria de Política de Saúde. Organização Panamericana da Saúde. Guia alimentar para crianças menores de 2 anos. Brasília: MS; 2002.

2. Monte CMG, Giugliani ERJ. Recomendações para alimentação complementar da criança em aleitamento materno. J Pediatr 2004; 80(5):131-141.

3. Organização Mundial da Saúde (OMS). Estratégia global para a alimentação de lactentes e crianças de primeira infância. São Paulo: IBFAN Brasil; 2005.

4. Sigulem DM, Taddei JAAC. Nutrição e alimentação nos dois primeiros anos de vida. São Paulo: Compacta Nutrição; 2004.

5. Vieira RW, Dias RP, Coelho SC, Ribeiro RL. Do aleitamento materno à alimentação complementar: atuação do profissional nutricionista. Saude \& Amb 2009; 4(2):1-8.

6. World Health Organization (WHO). Indicators for assessing infant and young child feeding practices part 3: country profiles. Geneva: WHO; 2010.

7. Ling L, Sprag D, Stein P, Myers ML Guidelines for diet counseling. J Am Diet Assoc 1975; 66(6):571-575.

8. Rodrigues EM, Soares FPTP, Boog MCF. Resgate do conceito de aconselhamento no contexto do atendimento nutricional. Rev Nutr 2005; 18(1):119-128.

9. Gabriel CG, Santos MV, Vasconcelos FAG. Avaliação de um programa para promoção de hábitos alimentares saudáveis em escolares de Florianópolis, Santa Catarina. Rev Bras Saúde Matern Infant 2008; 8(3):299-308.

10. World Health Organization (WHO). Strengthening action to improve feeding of infants and young children 6-23 months of age in nutrition and child health programmes: report of proceedings. Geneva: WHO; 2008.

11. Carvalho AP, Oliveira VB, Santos LC. Hábitos alimentares e práticas de educação nutricional: atenção a crianças de escola de Belo Horizonte, MG. Pediatr 2010; 32(1):20-27.

12. Davanço GM, Taddei JAAC, Gaglione CP. Conhecimentos, atitudes e práticas de professores de ciclo básico, expostos e não expostos a curso de educação nutricional. Rev Nutr 2004; 17(2):177-184.

13. Deminice R, Laus MF, Marins TM, Silveira SDO, Dutra-de-Oliveira JE. Impacto de um programa de educação alimentar sobre conhecimentos, práticas alimentares e estado nutricional de escolares. Alim Nutr 2007; 18(1):35-40.

14. Santos IS, Victora CG, Martines J, Gonçalves H, Gigante DP, Valle NJ, Pelto G. Avaliação da eficácia do aconselhamento nutricional dentro da estratégia do AIDPI (OMS/UNICEF). Rev Bras Epidemiol 2002; 5(1):15-29.

15. Bassichetto KC, Rea MF. Aconselhamento em alimentação infantil: um estudo de intervenção. $J P e$ diatr 2008; 84(1):75-82.

16. Rea MF, Venâncio SI. Avaliação do curso de aconselhamento em amamentação OMS/UNICEF. $J$ Pediatr 1999; 75(2):112-118.

17. Venancio SI, Monteiro CA. A tendência da prática da amamentação no Brasil nas décadas de 70 e 80 . Rev Bras Epidemiol 1998; 1(1):40-49.

18. Giugliani ERJ, Victora CG. Alimentação complementar. J Pediatr 2000; 76(3):253-262.
19. Bueno LGS, Teruya KM. Aconselhamento em amamentação e sua prática. J Pediatr 2004; 80(5):126130.

20. Ciconi RCV, Venâncio SI, Escuder MML. Avaliação dos conhecimentos de equipe do Programa Saúde da Família, sobre o manejo de aleitamento materno em um município da região metropolitana de São Paulo. Rev Bras Saúde Mater Infant 2004; 4(2):193-202.

21. Leite AM, Silva IA, Scochi CGS. Comunicação nãoverbal: uma contribuição para o aconselhamento em amamentação. Rev Lat Am Enfermagem 2004; 12(2):258-264.

22. Valle NJ, Santos IS, Gigante DP. Intervenções nutricionais e crescimento infantil em crianças de até dois anos de idade: uma revisão sistemática Cad Saude Publica 2004; 20(6):1458-1467.

23. Ferreira VA, Magalhães R. Nutrição e promoção da saúde: perspectivas atuais Cad Saude Publica 2007; 23(7):1674-1681.

24. Toma TS. Alimentação de crianças do Programa Saúde da Família (PSF): fatores associados à amamentação plena e impacto de um curso de aconselhamento em alimentação infantil nos conhecimentos de trabalhadores da saúde [tese]. São Paulo: Faculdade de Saúde Pública; 2008.

25. Vasconcelos ACCP, Pereira IDF, Cruz PJSC. Práticas educativas em nutrição na atenção básica em saúde: reflexões a partir de uma experiência de extensão popular em João Pessoa-Paraíba. Rev APS 2008; 11(3):334-340.

26. Arimatea JE, Castro LMC, Rotemberg S. Práticas Alimentares de Crianças Menores de um Ano: as orientações de profissionais de saúde e as recomendações do Ministério da Saúde. Ceres 2009; 4(2):65-78.

27. Costa ARC, Teodoro TN, Araújo MFM. Análise dos conhecimentos e da prática de profissionais de saúde na promoção e no apoio à amamentação: estudo de revisão. Comun Cienc Saude 2009; 20(1): 55-63.

28. Ferreira JV, Castro LMC, Menezes MFG. Alimentação no Primeiro Ano de Vida: a conduta dos profissionais de saúde e a prática exercida pela família. Ceres 2009; 4(3):117-129.

29. Bassichetto KC, Réa MF. Aconselhamento em alimentação infantil: contribuição para a discussão dos pressupostos teóricos. Saude Colet 2010; 42(7):189-194.

30. Dias MCP, Freire LMS, Franceschini SCC. Recomendações para alimentação complementar de crianças menores de dois anos. Rev Nutr 2010; 23(3): 475-486.

31. Silva CM, Basso DF, Locks A. Alimentação na primeira infância: abordagem para a promoção da saúde bucal. Rev Sul Bras Odontol 2010; 7(4):458-465.

32. Galvão DG. Formação em aleitamento materno e suas repercussões na prática clínica. Rev Bras Enferm 2011; 64(2):308-314. 
33. Susin LRO, Giugliani ERJ, Kummer SCC, Maciel M, Benjamin ACW, Machado DB, Barcaro M, Draghetti V. Uma estratégia simples que aumenta os conhecimentos das mães em aleitamento materno e melhora as taxas de amamentação. Rev Chil Pediatr 2000; 71(5):461-470.

34. Albernaz E, Victora CG. Impacto do aconselhamento face a face sobre a duração do aleitamento exclusivo. Rev Panam Salud Publica 2003; 14(1):1724.

35. Santos I, Cesar JA, Minten G, Marco PL, Valle N. Efetividade do aconselhamento nutricional da Pastoral da Criança sobre a variação de hemoglobina entre menores de seis anos de idade. Cad Saude Publica 2005; 21(1):130-140.

36. Braga DF, Machado MMT, Bosi MLM. Amamentação exclusiva de recém-nascidos prematuros: percepções e experiências de lactantes usuárias de um serviço público especializado. Rev Nutr 2008; 21(3):293-302.

37. Pelegrin RCP. O cuidado com a alimentação de crianças menores de um ano na perspectiva materna [dissertação]. São Paulo: Universidade de São Paulo; 2008.

38. Silva MB, Albernaz EP, Mascarenhas MLW; Silveira RB. Influência do apoio à amamentação sobre o aleitamento materno exclusivo dos bebês no primeiro mês de vida e nascidos na cidade de Pelotas, Rio Grande do Sul, Brasil. Rev Bras Saúde Mater Infant 2008; 8(3):275-284.

39. Salve JM, Silva IA. Representações sociais de mães sobre a introdução de alimentos complementares para lactentes. Acta paul enferm 2009; 22(1):43-48.

40. Bortolini GA, Fisberg M. Orientação nutricional do paciente com deficiência de ferro. Rev Bras Hematol Hemoter 2010; 32(2):105-113.

41. Moreira PVL, Freitas CHSM. Educação em saúde nos cenários de prática dos estudantes de nutrição. Rev APS 2010; 13(4):500-504.

42. Garcia MT, Granado FS, Cardoso MA. Alimentação complementar e estado nutricional de crianças menores de dois anos atendidas no Programa Saúde da Família em Acrelândia, Acre, Amazônia Ocidental Brasileira. Cad Saude Publica 2011; 27(2):305-316.

43. Verrall T, Gray-Donald K. Impact of a food-based approach to improve iron nutrition of at-risk infants in northern Canada. Prev Med 2005; 40(6):896-903.

44. World Health Organization (WHO), UNICEF. Breastfeeding counselling: A training course. Geneva: WHO, UNICEF; 1993.

45. World Health Organization (WHO). Infant and young child feeding counselling: an integrated course. Geneva: WHO; 2006.

46. Brasil. Ministério da Saúde (MS). ENPACS: Estratégia Nacional Para Alimentação Complementar Saudável: Caderno do Tutor / Ministério da Saúde, Rede Internacional em Defesa do Direito de Amamentar - IBFAN Brasil. Brasília: MS; 2010.

47. Pan American Health Organization (Opas), World Health Organization (WHO). Guiding principles for complementary feending of the breastfed child. Division of Health Promotion and Protection. Food and Nutrition Program. Washington, Geneva: Opas, WHO; 2003.
48. Barbosa RMS, Salles-Costa R, Soares EA. Guias alimentares para crianças: aspectos históricos e evolução. Rev Nutr 2006; 19(2):255-263.

49. Barbosa RMS, Colares LGT, Soares EA. Desenvolvimento de guias alimentares em diversos países. Rev Nutr 2008; 21(4):455-467.

50. Bassichetto KC, Rea MF, Donato AF. Atualização profissional em aconselhamento em alimentação infantil: uma experiência de avaliação. Bol Inst Saude 2009; (48):41-45.

51. Travassos C, Viacava F, Pinheiro R, Brito A. Utilização dos serviços de saúde no Brasil: gênero, características familiares e condição social. $\mathrm{Rev} \mathrm{Pa}$ nam Salud Publica 2002; 11(5-6):365-373.

52. Dias G, Franceschini SCC, Reis JRR, Reis RS, Siqueira-Batista R, Cotta RMM. A vida nos olhos, o coração nas mãos: concepções e representações femininas do processo saúde-doença. Hist Cienc Saude Manguinhos 2007; 14(3):779-800.

53. Costa GD, Cotta RMM, Reis JR, Ferreira MLSM, Reis RS, Franceschini SCC. Avaliação da atenção à saúde da criança no contexto da Saúde da Família no Município de Teixeiras, Minas Gerais. Cienc Saud Colet 2011; 16(7):3229-3240.

54. Correa EM, Corso ACT, Moreira EAM, Kazapi IAM. Alimentação complementar e características maternas de crianças menores de dois anos de idade em Florianópolis (SC). Rev Paul Pediatr 2009; 27(3): 258-264.

55. Romanelli G. O significado da alimentação na família: uma visão antropológica. Medic 2006; 39(3):333-339.

56. Roida S, Hassi A, Maoulainine FM, Aboussad A. Les pratiques de l'allaitement maternel à la maternité universitaire de Marrakech (Maroc). J Pediatr Pueric 2010; 23(2):70-75.

57. Oliveira LD, Giugliani ERJ, Santo LCE, Nunes LM. Impact of a strategy to prevent the introduction of non-breast milk and complementary foods during the first 6 months of life: A randomized clinical trial with adolescent mothers and grandmothers. Early Hum Dev 2012; 88(6):357-361.

58. Perosa GB, Carvalhaes MABL, Benício MHD, Silveira FCP. Estratégias alimentares de mães de crianças desnutridas e eutróficas: estudo qualitativo mediante observação gravada em vídeo. Cien Saude Colet 2011; 16(11):4445-4464.

59. Brasil. Ministério da Saúde (MS). Boletim ENPACS Brasília: MS; 2011.

60. Cotta RMM, Mendonça ET, Costa GD. Portfólios reflexivos: construindo competências para o trabalho no Sistema Único de Saúde. Rev Panam Salud Publica 2011; 30(5):415-421.

61. Macedo IC. Capacitação para educação nutricional dirigida a professores de um curso de educação infantil [dissertação]. São Paulo: Universidade de São Paulo; 2003.

62. Kerr FRM. A relação médico-paciente e a qualidade do atendimento médico. [dissertação]. São Paulo: Faculdade de Saúde Pública; 2000. 
63. Silva DO, Recine EGIG, Queiroz EFO. Concepções de profissionais de saúde da atenção básica sobre a alimentação saudável no Distrito Federal, Brasil. Cad Saude Publica 2002; 18(5):1367-1377.

64. Morgado CMC, Werneck GL, Hasselmann MH. Rede e apoio social e práticas alimentares de crianças no quarto mês de vida. Cien Saud Colet 2013; 18(2):367-376.

65. Gomes KO, Cotta RMM, Araújo RMA, Cherchiglia ML, Martins TCP. Atenção Primária à Saúde - a 'menina dos olhos' do SUS: sobre as representações sociais dos protagonistas do Sistema Único de Saúde. Cien Saud Colet 2011; 16(1):881-892.

66. Panigassi G. Profissionais de saúde: conhecimento e conduta em aleitamento materno [dissertação]. Campinas: Faculdade de Ciências Médicas; 2000.

67. Boog MCF. Dificuldades encontradas por médicos e enfermeiros na abordagem de problemas alimentares. Rev Nutr 1999; 12(3):261-272.

68. Ayres JRCM. Sujeito, intersubjetividade e práticas de saúde. Cien Saude Colet 2001; 6(1):63-72.

69. World Health Organization (WHO). Complementary feeding: report of the global consultation, and summary of guiding principles for complementary feeding of the breastfed child. Geneva: WHO; 2002.

70. Mennella JA, Turnbull B, Ziegler PJ, Martinez H. Infant feeding practices and early flavor experiences in Mexican infants: an intra-cultural study. $J \mathrm{Am}$ Diet Assoc 2005; 105(6):908-915.

71. Ziegler P, Hanson C, Ponza M, Novak T, Hendricks K. Feeding Infants and Toddlers Study: Meal and Snack Intakes of Hispanic and Non-Hispanic Infants and Toddlers. J Am Diet Assoc 2006; 106(1):107-123.

72. Schwartz C, Chabanet C, Lange C, Issanchou S, Nicklaus S. The role of taste in food acceptance at the beginning of complementary feeding. Physiol Behav 2011; 104(4):646-652.

Artigo apresentado em 20/06/2012

Aprovado em 31/07/2012

Versão final apresentada em 20/08/2012 